\title{
Synthesis and characterization of new diiodocoumarin derivatives with promising antimicrobial activities
}

\author{
Hany M. Mohamed ${ }^{1,2}$, Ashraf H. F. Abd EL-Wahab ${ }^{* 1,3}$, \\ Ahmed M. EL-Agrody ${ }^{4}$, Ahmed H. Bedair ${ }^{1}$, Fathy A. Eid ${ }^{1}$, \\ Mostafa M. Khafagy ${ }^{1}$ and Kamal A. Abd-EL-Rehem ${ }^{1}$
}

\section{Full Research Paper}

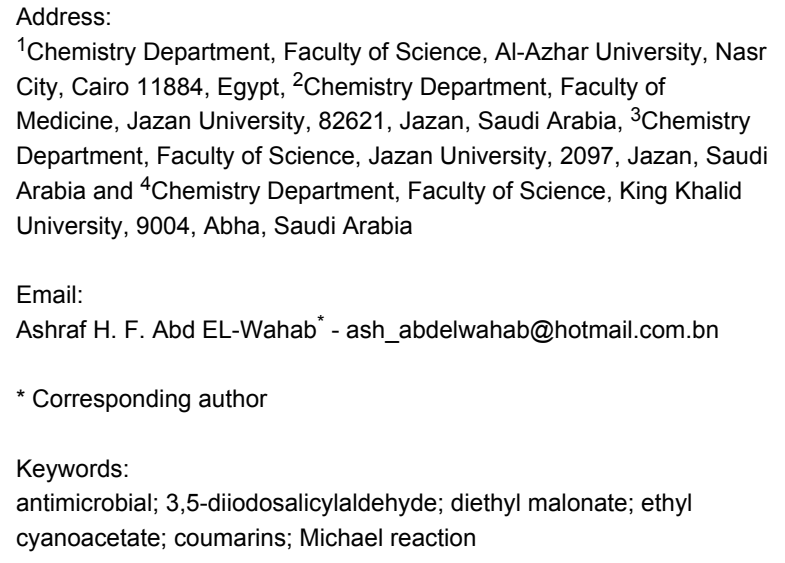

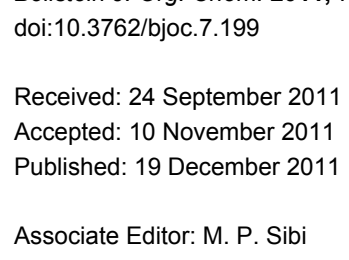

\begin{abstract}
A series of 6,8-diiodocoumarin-3- $N$-carboxamides (4-11) were prepared. Treatment of ethyl 6,8-diiodocoumarin-3-carboxylate (1) with ethyl cyanoacetate/ $\mathrm{NH}_{4} \mathrm{OAc}$ gave ethyl 2-(3-carbamoyl-6,8-diiodocoumarin-4-yl)-2-cyanoacetate (12) and 2-amino-4hydroxy-7,9-diiodocoumarino[3,4-c]pyridine-1-carbonitrile (13), and treatment with acetone in the presence of $\mathrm{NH}_{4} \mathrm{OAc}$ or methylamine gave the ethyl 4-oxo-2,6-methano-2-methyl-3,4,5,6-tetrahydro-8,10-diiodobenzo[2,1-g]-2H-1,3-oxazocine-5-carboxylate derivatives 14a,b. All compounds were evaluated for their antimicrobial activity and the compounds 12-14a,b exhibited a pronounced effect on all tested microorganisms.
\end{abstract}

\section{Introduction}

Coumarins and their derivatives are biologically and pharmaceutically interesting compounds known for their use as additives in food, perfumes, cosmetics, pharmaceuticals, platelet aggregation and agrochemicals $[1,2]$. Coumarins have also been reported to exhibit several biological activities, such as antimicrobial, anticancer, antifungal, anti-HIV and antioxidant properties [3-6], and they also served as versatile precursors for many organic transformations in the synthesis of a number of 
drug-like molecules [7,8]. Moreover, coumarin-based dyes and pigments are organic fluorescent materials exhibiting unique photochemical and photophysical properties, which render them useful in a variety of applications such as dye lasers, anion sensors, organic light-emitting diodes and solar cells $[9,10]$.

Iodo-organic derivatives have been widely used as diagnosticimaging drugs (such as diatrizoate meglumine, diatrizoic acid, iodipamide, iodixanol, iohexol, iomeprol and iopamidol) and as amebicides $[11,12]$. The benzoxazocine derivatives have received considerable attention due to their pharmacological properties, such as their antidepressant, antithrombotic, antipsychotic (for the central nervous system, CNS) and anti-breastcancer activities [13].

In view of the important biological properties of the diiodocoumarin derivatives and iodo-organic compounds as medical agents, we planned to synthesize some new diiodocoumarin derivatives bearing side chains with different structures, as such derivatives could possess interesting and useful biological properties.

\section{Results and Discussion}

Interaction of 3,5-diiodosalicylaldehyde with diethyl malonate according to the literature procedure $[14,15]$ afforded ethyl 6,8 - diiodocoumarin-3-carboxylate (1). Treatment of $\mathbf{1}$ with hot ethanolic $\mathrm{KOH}(10 \%)$ followed by acidification with $\mathrm{HCl}$ gave the corresponding 6,8-diiodocoumarin-3-carboxylic acid (2), which on treatment with $\mathrm{SOCl}_{2}$ gave the 6,8-diiodocoumarin-3carbonyl chloride (3). Treatment of $\mathbf{1}$ with piperidine in boiling ethanol or with $p$-phenylenediamine in boiling $\mathrm{AcOH}$ afforded the 6,8-diiodocoumarin-3-carboxamide derivatives $\mathbf{4}$ and $\mathbf{5}$, respectively. Interaction of $\mathbf{3}$ with glycine in dry benzene under reflux gave the new 6,8-diiodocoumarin-3- $N, N$-dimethylcarboxamide (7) instead of 6,8-diiodocoumarin-3-ylcarbonylglycine (6). The formation of compound 7 suggests that two glycine molecules react with 1 followed by the loss of ammonia and decarboxylation, furnishing the observed product (Scheme 1).

The structures of compounds $\mathbf{3}-\mathbf{5}$ and $\mathbf{7}$ were confirmed by IR, ${ }^{1} \mathrm{H}$ NMR, ${ }^{13} \mathrm{C}$ NMR and MS. The IR spectra for compound $\mathbf{3}$ showed 1774, $1718 \mathrm{~cm}^{-1}$ (2 CO); for compound 4 1713, 1631 $\mathrm{cm}^{-1}$ (2 CO); for compound $51718 \mathrm{~cm}^{-1}$ (CO); and for compound 7 1722, $1635 \mathrm{~cm}^{-1}$ (2 CO). ${ }^{1} \mathrm{H}$ NMR for compounds 3-5 and 7 showed $\delta$ at $7.64-8.70 \mathrm{ppm}(\mathrm{s}, 1 \mathrm{H}, \mathrm{H}-4)$, and ${ }^{13} \mathrm{C} \mathrm{NMR}$ for compounds 3 and $\mathbf{5}$ showed $\delta$ at 143.2 and 147.2 ppm (C-4), respectively. The mass spectra of compounds 3 and 7 showed the corresponding molecular ion peaks at $m / z 460\left(\mathrm{M}^{+}, 2.6 \%\right)$ and $m / z 469\left(\mathrm{M}^{+}, 18.5 \%\right)$. The fragmentation pattern of compounds 3 and 7 are illustrated in Scheme 2.<smiles>O=Cc1cc(I)cc(I)c1O</smiles><smiles>CCOc1cc(I)c2oc(=O)c(C(=O)O)cc2c1</smiles><smiles>O=C(O)c1cc2cc(I)cc(I)c2oc1=O</smiles><smiles>C/C=C(/N)CCc1cc2cc(I)cc(I)c2oc1=O</smiles><smiles>O=C(Cl)c1cc2cc(I)cc(I)c2oc1=O</smiles><smiles>O=C(c1cc2cc(I)cc(I)c2oc1=O)N1CCCCC1</smiles>
3<smiles>CN(C)C(=O)c1cc2cc(I)cc(I)c2oc1=O</smiles>
$\mathrm{NH}_{2} \mathrm{CH}_{2} \mathrm{COOH}$<smiles>CC(C)(C)CC(C)(C)C(=O)c1cc2cc(I)cc(I)c2oc1=O</smiles> 


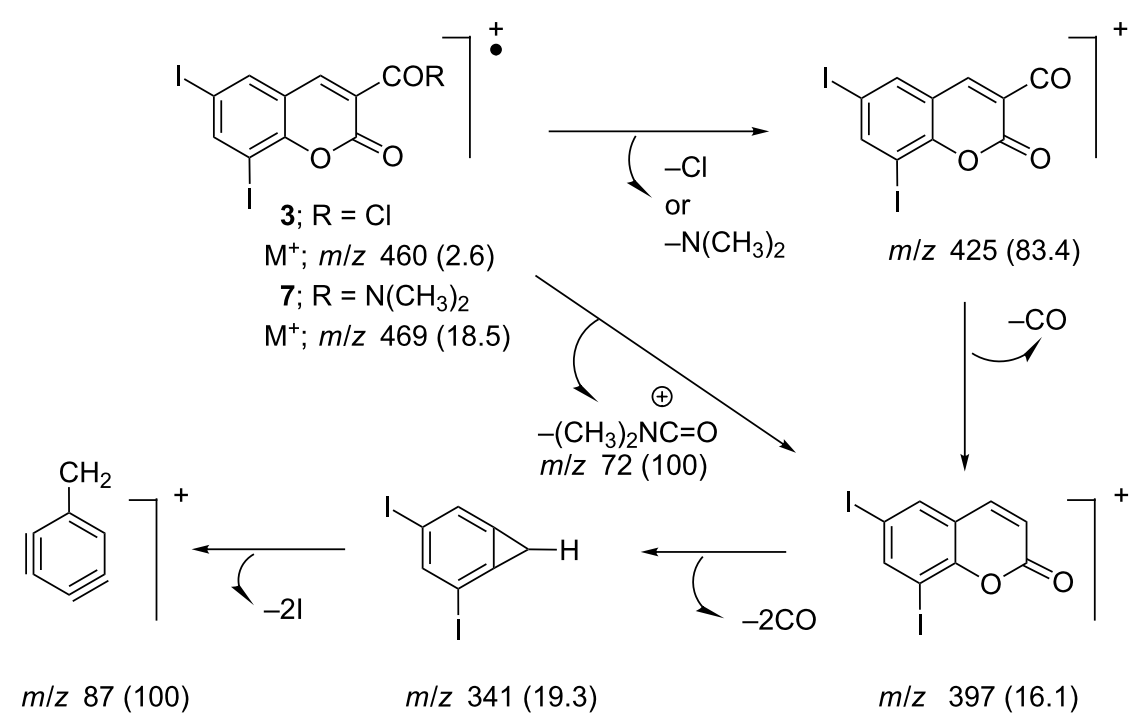

Scheme 2: Proposed fragmentation pathways for the El ions of the substituted 6,8-diiodocoumarins 3 and 7.

Reactions of 3 with 4-aminophenylethanol or $p$-aminophenol, or with potentially bifunctional amino acids (anthranilic acid and $p$-aminophenylacetic acid), was successful, and the corresponding 6,8-diiodocoumarin-3-carboxamide derivatives 8-11 were obtained (Scheme 3 ).
The structures of compounds 8-11 were established by IR, ${ }^{1} \mathrm{H}$ NMR, ${ }^{13} \mathrm{C}$ NMR and MS. The IR spectra of compound $\mathbf{8}$ showed $3287 \mathrm{~cm}^{-1}(\mathrm{OH}, \mathrm{NH})$ and $1719 \mathrm{~cm}^{-1}(\mathrm{CO})$ and for compound $93217 \mathrm{~cm}^{-1}(\mathrm{NH}, \mathrm{OH})$ and $1720 \mathrm{~cm}^{-1}$ (CO). ${ }^{1} \mathrm{H}$ NMR for 8 showed $\delta$ at $3.01\left(\mathrm{t}, J=7.0 \mathrm{~Hz}, 2 \mathrm{H}, \mathrm{Ar}-\mathrm{CH}_{2}\right)$,

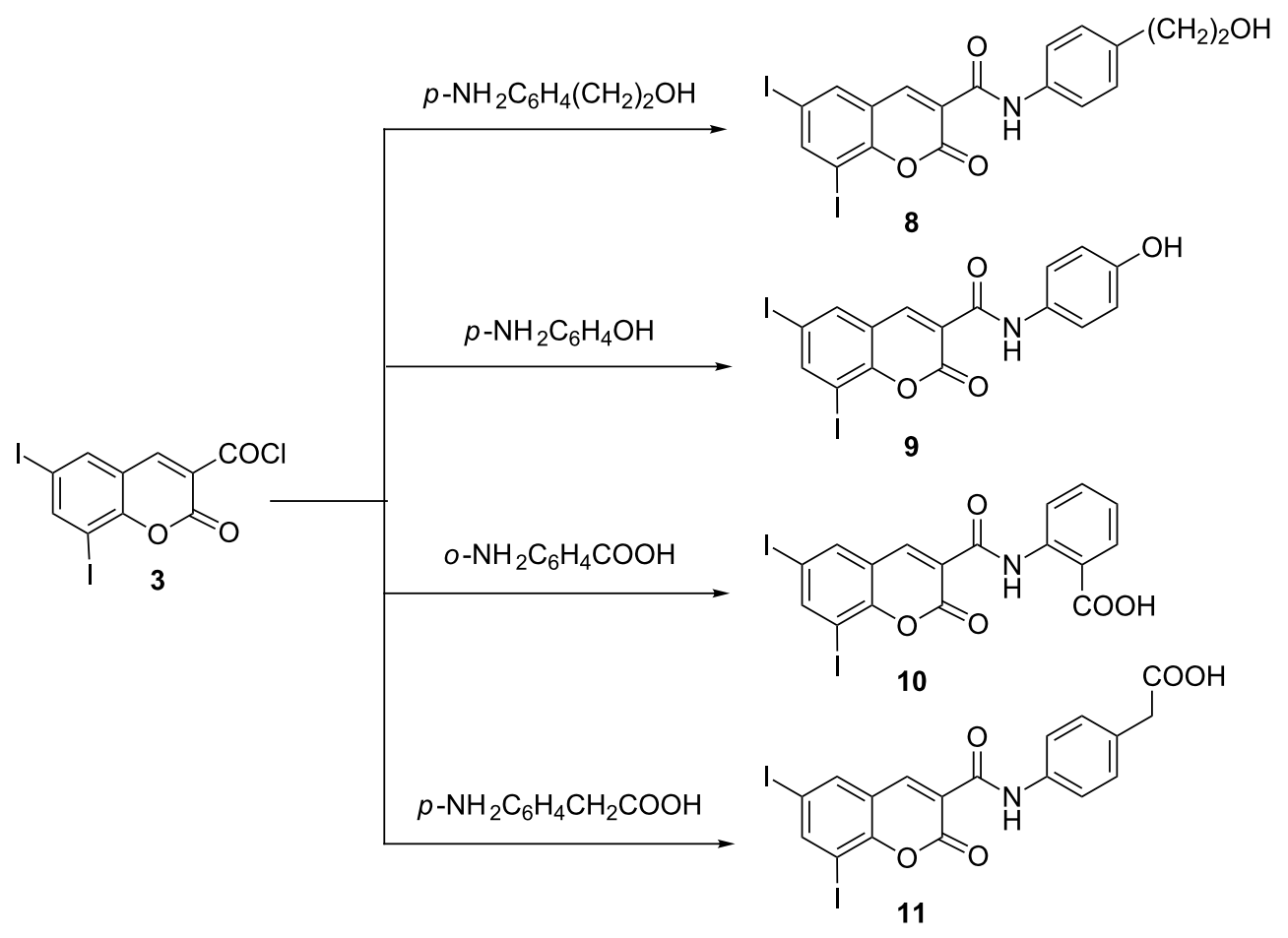

Scheme 3: Synthesis of 6,8-diiodocoumarin-3- $N$-carboxamide derivatives 8-11. 
$3.56(\mathrm{~s}, 1 \mathrm{H}, \mathrm{OH}), 3.83\left(\mathrm{t}, J=7.0 \mathrm{~Hz}, 2 \mathrm{H}, \mathrm{CH}_{2}-\mathrm{OH}\right)$, and $10.49 \mathrm{ppm}(\mathrm{s}, 1 \mathrm{H}, \mathrm{NH})$, and for compound 11 at $3.55(\mathrm{~s}, 2 \mathrm{H}$, $\left.\mathrm{CH}_{2}\right), 8.71$ (s, $\left.1 \mathrm{H}, \mathrm{H}-4\right), 10.10$ (brs, $\left.1 \mathrm{H}, \mathrm{NH}\right)$, and 10.49 (s, $1 \mathrm{H}$, $\mathrm{OH}$ ). The ${ }^{13} \mathrm{C}$ NMR for 11 showed $\delta$ at 160 (CO $\delta$ lactone), $163.4(\mathrm{CONH})$, and $176.5 \mathrm{ppm}(\mathrm{COOH})$. The mass spectra of compounds 8-11 provided additional evidence for the proposed structures.

As the C3-C4 olefinic bond in ethyl 6,8-diiodocoumarin-3carboxylate (1) is activated by conjugation with electron-withdrawing carbonyl groups, the behavior of $\mathbf{1}$ towards activated methylene compounds under Michael reaction conditions was investigated. Thus, treatment of $\mathbf{1}$ with ethyl cyanoacetate/ $\mathrm{NH}_{4} \mathrm{OAc}$ in boiling ethanol afforded two reaction products. The insoluble reaction product was identified as ethyl 2-(3carbamoyl-6,8-diiodocoumarin-4-yl)-2-cyanoacetate (12) and the soluble reaction product was identified as 2-amino-4hydroxy-7,9-diiodocoumarino[3,4-c]pyridine-1-carbonitrile (13), which probably formed as a result of amide formation, dehydration and intramolecular cyclization (Scheme 4).

The structures of compounds $\mathbf{1 2}$ and $\mathbf{1 3}$ were established by IR, ${ }^{1} \mathrm{H}$ NMR, ${ }^{13} \mathrm{C}$ NMR and MS. The IR spectra of compound 12 showed 3309, $3277 \mathrm{~cm}^{-1}\left(\mathrm{NH}_{2}\right), 2206 \mathrm{~cm}^{-1}(\mathrm{CN})$, and
$1643 \mathrm{~cm}^{-1}$ (CO), while the ${ }^{1} \mathrm{H}$ NMR for compound 13 showed $\delta$ at 7.89 (brs, $2 \mathrm{H}, \mathrm{NH}_{2}$ ), and 9.06 (brs, $\left.1 \mathrm{H}, \mathrm{OH}\right)$. The spectral data of compound $\mathbf{1 3}$ confirmed its enol structure.

Reaction of $\mathbf{1}$ with acetone in the presence of $\mathrm{NH}_{4} \mathrm{OAc}$ or methylamine at room temperature for 7 days gave 1,3-oxazocine-5-carboxylate derivatives (14a,b) [16-18] (Scheme 5). The formation of $\mathbf{1 4}$ indicates that the activated methylene compounds attack at the $\mathrm{C} 3-\mathrm{C} 4$ olefinic bond in $\mathbf{1}$ under Michael reaction conditions to yield a cyclic Michael adduct, which underwent hydrolysis by $\mathrm{NH}_{3}$ or $\mathrm{MeNH}_{2}$ and cyclization through the elimination of $\mathrm{H}_{2} \mathrm{O}$ (Scheme 5).

The structure of compound 14a was established by ${ }^{13} \mathrm{C}$ NMR, which showed $\delta$ at $42.5\left(\mathrm{CH}_{2}(\mathrm{c})\right), 168.4 \mathrm{~cm}^{-1}$ (CONH), and $170 \mathrm{~cm}^{-1}$ (CO). The structures of all newly synthesized compounds were confirmed by IR, ${ }^{1} \mathrm{H}$ NMR, ${ }^{13} \mathrm{C}$ NMR and mass spectrometry.

The inhibitory effects of the synthetic compounds against these organisms are given in Table 1, Figure 1 and Figure 2. Among the series tested, compounds 12-14a,b exhibited excellent antibacterial activity, better than the standard ampicillin, against two species of Gram-positive bacteria, Staphylococcus aureus<smiles></smiles><smiles>CCOC(=O)C(c1c(C(N)=O)c(=O)oc2c(I)cc(I)cc12)C(C)C</smiles>

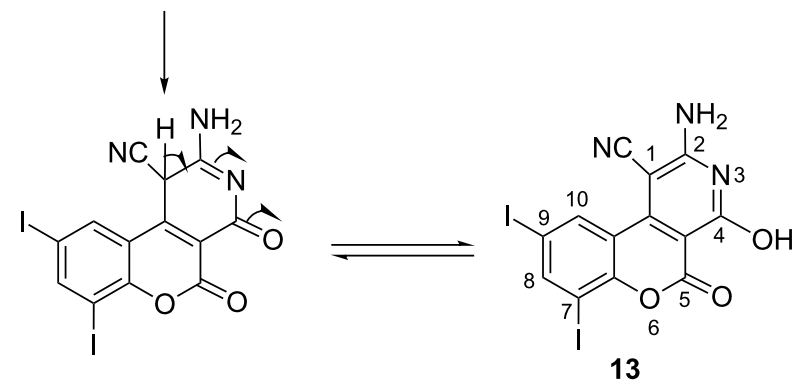




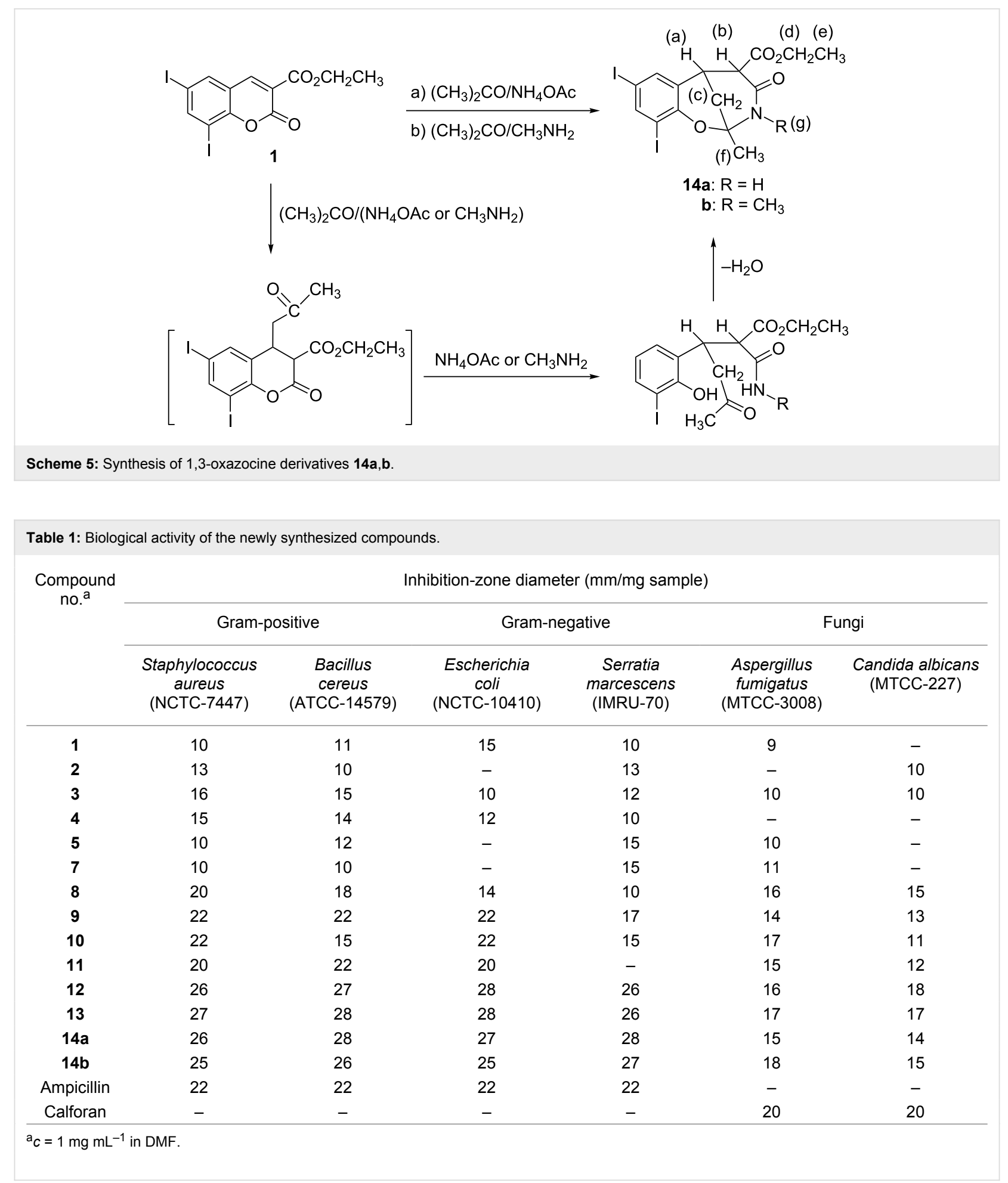

(NCTC-7447), Bacillus cereus (ATCC-14579) and two Gramnegative bacteria, Escherichia coli (NCTC-10410) and Serratia marcescens (IMRU-70), while the same compounds showed moderate antifungal activity against the tested organisms. Compounds 9-11 exhibited comparable activity to ampicillin against the tested bacteria and moderate to weak antifungal activity against the tested organisms. Furthermore, compounds 1-8 showed moderate to weak activities against all the tested bacteria and fungi, compared with the standards ampicillin and calforan. In addition, compounds $\mathbf{2}$ and $\mathbf{5}$ in the series were found to be inactive against Escherichia coli (NCTC-10410), while compound $\mathbf{1 1}$ was inactive against Serratia marcescens 


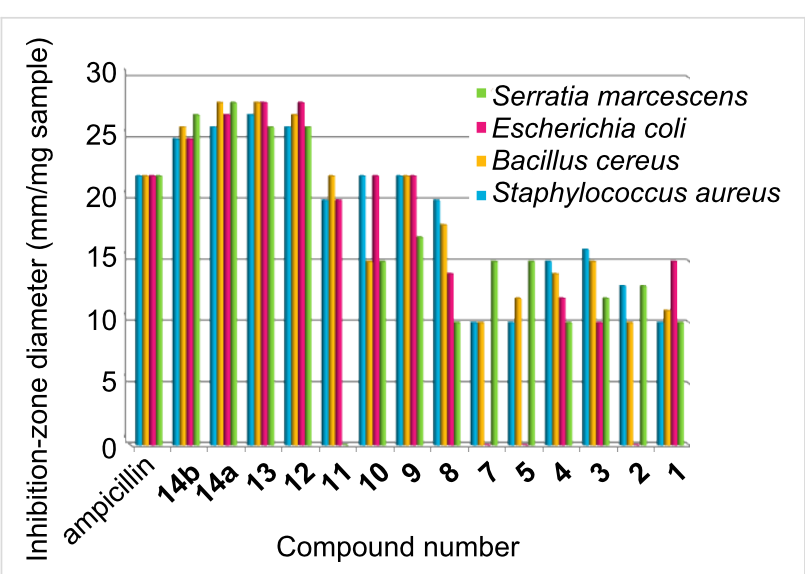

Figure 1: Graphical representation of the antibacterial activity of tested compounds compared to ampicillin.

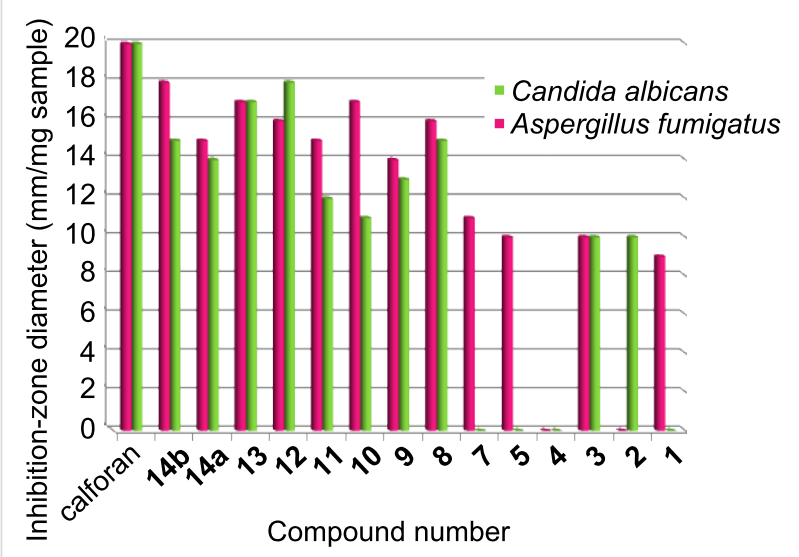

Figure 2: Graphical representation of antifungal activity of tested compounds, compared to calforan.

(IMRU-70). An investigation of the structure-activity relationship (SAR) revealed that the activity is considerably affected by the presence of the diiodocoumarino[3,4-c]pyridine, 2-methyl8,10-diiodobenzo[2,1-g]-2H-1,3-oxazocine, diiodocoumarin-3carboxamide or 2,3-dimethyl-8,10-diiodobenzo[2,1-g]-2H-1,3oxazocine, and slightly decreases with the presence of different amide groups at position $\mathrm{C}-3$ of the diiodocoumarin moiety or with the presence of ester, acid or acid chloride at position C-3 of the diiodocoumarin moiety.

\section{Experimental}

\section{General methods}

Melting points were determined on a Stuart melting point apparatus and are uncorrected; IR spectra were recorded in $\mathrm{KBr}$ on a FT-IR 5300 spectrometer and Perkin Elmer spectrum RXIFTIR system $\left(v, \mathrm{~cm}^{-1}\right)$. The ${ }^{1} \mathrm{H}$ NMR spectra at $300 \mathrm{MHz}$ and ${ }^{13} \mathrm{C}$ NMR spectra at $75 \mathrm{MHz}$ were recorded in $\mathrm{CDCl}_{3}$ or DMSO- $d_{6}$ on a Varian Mercury VX-300 NMR spectrometer.
Chemical shifts $(\delta)$ are related to that of the solvent. Mass spectra were measured on a Shimadzu GMMS-QP-1000 EX mass spectrometer at $70 \mathrm{eV}$. The elemental analyses were performed at the Microanalytical Center, Cairo University, Cairo (Egypt).

Ethyl 6,8-diiodocoumarin-3-carboxylate (1). Ethyl 6,8diiodocoumarin-3-carboxylate (1) was prepared by the interaction of 3,5-diiodosalicylaldehyde with diethyl malonate according to the literature procedures [19-21].

6,8-Diiodocoumarin-3-carboxylic acid (2). A solution of compound $1(0.47 \mathrm{~g}, 10 \mathrm{mmol})$ in absolute ethanol $(20 \mathrm{~mL})$ was mixed with ethanolic solution of $\mathrm{KOH}(10 \%)$, which was then refluxed for $10 \mathrm{~min}$. The reaction mixture was poured onto ice, acidified with $\mathrm{HCl}$ and recrystallized from ethanol [22].

6,8-Diiodocoumarin-3-carbonyl chloride (3). Compound 2 $(0.44 \mathrm{~g}, 10 \mathrm{mmol})$ was dissolved in dry benzene $(40 \mathrm{~mL}), 2 \mathrm{~mL}$ of thionyl chloride was added and the solution was refluxed for $1 \mathrm{~h}$. A few drops of formic acid were added to eliminate the unreacted thionyl chloride, and the solvent was removed under reduced pressure. The solid obtained was recrystallized from benzene. Yellow crystals: Yield $92 \%$; mp $180{ }^{\circ} \mathrm{C}$; Anal. calcd for $\mathrm{C}_{10} \mathrm{H}_{3} \mathrm{ClI}_{2} \mathrm{O}_{3}$ : C, 26.10; $\mathrm{H}, 0.65$; found: $\mathrm{C}, 26.11 ; \mathrm{H}, 0.67$; IR (KBr, cm $\left.{ }^{-1}\right)$ : 3055 (C-H aromatic), 1774, 1718 (2 CO); ${ }^{1} \mathrm{H}$ NMR $\left(300 \mathrm{MHz}, \mathrm{CDCl}_{3}, \delta / \mathrm{ppm}\right) 8.01(\mathrm{~d}, J=1.8 \mathrm{~Hz}, 1 \mathrm{H}$, Ar-H-7), 8.44 (d, $J=1.8 \mathrm{~Hz}, 1 \mathrm{H}, \mathrm{Ar}-\mathrm{H}-5), 8.58$ (s, 1H, H-4); ${ }^{13} \mathrm{C}$ NMR $\left(75 \mathrm{MHz}, \mathrm{CDCl}_{3}, \delta / \mathrm{ppm}\right) 86.0$ (C-6), 89.1 (C-8), 118.6 (C-3), 120.3 (C-4a), 138.2 (C-5), 147.2 (C-4), 149.4 (C-7), 153.6 (C-8a), 155.0 (CO $\delta$ lactone), 162.9 (CO); MS m/z (\% relative intensity): $460\left(\mathrm{M}^{+}, 2.6\right), 459(\mathrm{M}-1,30.4), 425$ (83.4), 341 (19.3), 214 (10.9), 87 (100).

6,8-Diiodo-3-(piperidine-1-carbonyl)coumarin (4). A solution of compound $1(0.47 \mathrm{~g}, 10 \mathrm{mmol})$ in absolute ethanol $(30 \mathrm{~mL})$ was refluxed with piperidine $(0.9 \mathrm{~g}, 10 \mathrm{mmol})$ for $1 \mathrm{~h}$. After cooling, the solid formed was filtered off, washed with ethanol and dried under vacuum. The solid obtained was recrystallized from benzene. Colorless crystals: Yield 80\%; mp $230{ }^{\circ} \mathrm{C}$; Anal. calcd for $\mathrm{C}_{15} \mathrm{H}_{13} \mathrm{I}_{2} \mathrm{NO}_{3}$ : C, 35.37; $\mathrm{H}, 2.55 ; \mathrm{N}$, 2.75; found: C, 35.36; H, 2.53; N, 2.76; IR $\left(\mathrm{KBr}, \mathrm{cm}^{-1}\right): 3040$ (C-H aromatic), 2935, 2854 (C-H aliphatic), 1713, 1631 (CO); ${ }^{1} \mathrm{H}$ NMR (300 MHz, $\left.\mathrm{CDCl}_{3}, \delta / \mathrm{ppm}\right) 1.59,1.67,3.29,3.69$ (m, $\left.10 \mathrm{H},\left(\mathrm{CH}_{2}\right)_{5}\right), 7.64$ (s, 1H, H-4), 7.78 (d, J=2.1 Hz, 1H, Ar-H7), $8.28(\mathrm{~d}, J=2.1 \mathrm{~Hz}, 1 \mathrm{H}, \mathrm{Ar}-\mathrm{H}-5) ;{ }^{13} \mathrm{C} \mathrm{NMR}(75 \mathrm{MHz}$, $\left.\mathrm{CDCl}_{3}, \delta / \mathrm{ppm}\right) 24.30,25.40,48.03\left(\mathrm{CH}_{2}\right.$ piperidine $), 86.0$ (C-8), 89.1 (C-6), 118.6 (C-3), 120.3 (C-4a), 138.2 (C-5), 147.2 (C-4), 149.4 (C-7), 153.6 (C-8a), 155.0 (CO $\delta$ lactone), 162.9 (CO-amide); MS $m / z$ (\% relative intensity): $509\left(\mathrm{M}^{+}, 0.3\right), 424$ (3.4), 341 (2.7), 214 (1.5), 84 (100). 
$\mathrm{N}$-(4-Acetamidophenyl)-6,8-diiodocoumarin-3-carboxamide (5). A solution of compound $1(0.47 \mathrm{~g}, 10 \mathrm{mmol})$ in glacial acetic acid $(30 \mathrm{~mL})$ was refluxed with $p$-phenylenediamine $(1.10 \mathrm{~g}, 10 \mathrm{mmol})$ for $2 \mathrm{~h}$. After cooling, the solid formed was filtered off, washed with ethanol, dried under vacuum and recrystallized from benzene. Colorless crystals: Yield 87\%; mp $319{ }^{\circ} \mathrm{C}$; Anal. calcd for $\mathrm{C}_{18} \mathrm{H}_{12} \mathrm{I}_{2} \mathrm{~N}_{2} \mathrm{O}_{4}$ : C, 37.64; H, 2.90; N, 4.88; found: C, 37.65; H, 2.92; N, 4.90; IR $\left(\mathrm{KBr}, \mathrm{cm}^{-1}\right): 3285$ (NH), 1718 (CO); ${ }^{1} \mathrm{H}$ NMR (300 MHz, DMSO- $\left.d_{6}, \delta / \mathrm{ppm}\right) 2.40$ (s, 3H, $\left.\mathrm{CH}_{3}\right), 7.28,7.66$ (2d, 4H, $\left.J=8.4 \mathrm{~Hz}, \mathrm{AB}-\mathrm{q}, \mathrm{Ar}-\mathrm{H}\right), 8.37$ (d , $J=1.8 \mathrm{~Hz}, 1 \mathrm{H}, \mathrm{Ar}-\mathrm{H}-7), 8.46$ (d , $J=1.8 \mathrm{~Hz}, 1 \mathrm{H}, \mathrm{Ar}-\mathrm{H}-5)$, 8.70 (s, $1 \mathrm{H}, \mathrm{H}-4), 8.90$ (s, $\left.1 \mathrm{H}, \mathrm{CH}_{3} \mathrm{CONH}\right), 10.12$ (brs, $1 \mathrm{H}$, $\mathrm{NH}) ;{ }^{13} \mathrm{C}$ NMR $\left(75 \mathrm{MHz}, \mathrm{DMSO}-d_{6}, \delta / \mathrm{ppm}\right) 23.1\left(\mathrm{CH}_{3}\right), 89.0$ (C-6), 90.1 (C-8), 121.5, 128.0 (C-2', 3', 5', 6'), 114.3, 133.5, 135.6 (C-3, 1', 4'), 125.3 (C-4a), 136.2 (C-5), 143.2 (C-4), 146.4 (C-7), 148.6 (C-8a), 160 (CO $\delta$ lactone), 163.7 (CO-amide), $170.0\left(\mathrm{COCH}_{3}\right) ; \mathrm{MS} m / z$ (\% relative intensity): $574\left(\mathrm{M}^{+}, 3\right)$, $532\left(\mathrm{M}-\mathrm{CH}_{2}=\mathrm{C}=\mathrm{O}, 38.7\right), 424$ (18.2), 341 (33.8), 298 (6.5), 171 (9.3), 107 (100).

6,8-Diiodocoumarin-3-N,N-dimethylcarboxamide (7). A solution of compound $3(0.46 \mathrm{~g}, 1 \mathrm{mmol})$ in dry benzene $(50 \mathrm{~mL})$ was refluxed with glycine $(0.75 \mathrm{~g}, 10 \mathrm{mmol})$ for $2 \mathrm{~h}$. After cooling, the solid formed was filtered off, washed with ethanol, dried under vacuum, and recrystallized from dioxane. Colorless crystals: Yield 83\%; mp $302{ }^{\circ} \mathrm{C}$; Anal. calcd for $\mathrm{C}_{12} \mathrm{H}_{9} \mathrm{I}_{2} \mathrm{NO}_{3}$ : C, 30.70; H, 1.92; N, 2.98; found: C, 30.72; H, 1.94; N, 3.00; IR ( $\mathrm{KBr}, \mathrm{cm}^{-1}$ ): 2931 (C-H aliphatic), 1722 and 1635 (CO); ${ }^{1} \mathrm{H}$ NMR (300 MHz, DMSO- $\left.d_{6}, \delta / \mathrm{ppm}\right) 2.93$ (s, $\left.3 \mathrm{H}, \mathrm{N}-\mathrm{CH}_{3}\right), 2.97$ (s, 3H, N-CH $), 8.02$ (s, 1H, H-4), 8.13 (d, $J$ $=2.1 \mathrm{~Hz}, 1 \mathrm{H}, \mathrm{Ar}-\mathrm{H}-7), 8.38(\mathrm{~d}, J=2.1 \mathrm{~Hz}, 1 \mathrm{H}, \mathrm{Ar}-\mathrm{H}-5)$; MS $m / z$ (\% relative intensity): $469\left(\mathrm{M}^{+}, 18.5\right), 425$ (83.4), 397 (16.1), 341 (19.3) and 72 (100).

\section{General procedure for the synthesis of 6,8- diiodocoumarin-3-carboxamide derivatives \\ 8-11}

To a well-stirred solution of $3(0.46 \mathrm{~g}, 1 \mathrm{mmol})$ in dry dichloromethane (DCM) containing a few drops of triethylamine (TEA) an equivalent amount of an ambient nucleophile [4-aminophenylethanol, $p$-aminophenol, anthranilic acid and $p$-aminophenylacetic acid $(1.2 \mathrm{mmol})]$ was added. The reaction mixture was stirred at room temperature under dry conditions for $3 \mathrm{~h}$. DCM was removed under reduced pressure until dryness, the obtained solid was then washed with $10 \% \mathrm{HCl}$ and the remaining solid recrystallized from dioxane.

N-(4-(2-Hydroxyethyl)phenyl)-6,8-diiodocoumarin-3carboxamide (8). Yellow crystals: Yield 87\%; mp $291{ }^{\circ} \mathrm{C}$; Anal. calcd for $\mathrm{C}_{18} \mathrm{H}_{13} \mathrm{I}_{2} \mathrm{NO}_{4}$ : C, 38.51; H, 2.32; N, 2.50; found: C, 38.52; H, 2.34; N, 2.51; IR $\left(\mathrm{KBr}, \mathrm{cm}^{-1}\right): 3287(\mathrm{OH}$,
NH), 3049 (Ar-H), 2958, 2928 (aliphatic-H), 1719 (CO); ${ }^{1} \mathrm{H}$ NMR (300 MHz, DMSO- $\left.d_{6}, \delta / \mathrm{ppm}\right) 3.01$ (t, $J=7.0 \mathrm{~Hz}, 2 \mathrm{H}$, $\left.\mathrm{Ar}-\mathrm{CH}_{2}\right), 3.56$ (s, 1H, OH), 3.83 (t, $\left.J=7.0 \mathrm{~Hz}, 2 \mathrm{H}, \mathrm{CH}_{2}-\mathrm{OH}\right)$, $7.29,7.63(2 \mathrm{~d}, J=8.2 \mathrm{~Hz}, 4 \mathrm{H}, \mathrm{AB}-\mathrm{q}, \mathrm{Ar}-\mathrm{H}), 8.37$ (d, $J=2.0$ $\mathrm{Hz}, 1 \mathrm{H}, \mathrm{H}-7), 8.46$ (d, $J=2.0 \mathrm{~Hz}, 1 \mathrm{H}, \mathrm{H}-5), 8.71$ (s, 1H, H-4), 10.49 (s, $1 \mathrm{H}, \mathrm{NH}) ;{ }^{13} \mathrm{C}$ NMR (75 MHz, DMSO- $\left.d_{6}, \delta / \mathrm{ppm}\right) 38.4$ $\left(\mathrm{Ar}-\mathrm{CH}_{2}\right), 62.2\left(\mathrm{CH}_{2}-\mathrm{OH}\right), 111.2,114.0(\mathrm{C}-6,8), 114.5,119.8$ $\left(\mathrm{C}-3^{\prime}, 2^{\prime}, 5^{\prime}, 6^{\prime}\right), 129.3,132.1$ (C-5,7), 135.4, 135.9, 136.0 (C-3,1',4'), 148.3 (C-4), 156.4, 159.9 (C4a,8a), 161.4 (CO $\delta$ lactone), 163.9 (CO-amide); $\mathrm{MS} m / z$ (\% relative intensity): 561 $\left(\mathrm{M}^{+}, 0\right), 543\left(\mathrm{M}-\mathrm{H}_{2} \mathrm{O}, 3\right), 530$ (67), $425\left(\mathrm{M}-\mathrm{NH}_{-} \mathrm{C}_{6} \mathrm{H}_{4}-\right.$ $\left.\mathrm{CH}_{2} \mathrm{CH}_{2} \mathrm{OH}, 100\right), 341$ (6), 107 (36), 128 (23), 127 (14), 87 (36).

$\mathrm{N}$-(4-Hydroxyphenyl)-6,8-diiodocoumarin-3-carboxamide (9). Yellow crystal: Yield $85 \%$; mp $303{ }^{\circ} \mathrm{C}$; Anal. calcd for $\mathrm{C}_{16} \mathrm{H}_{9} \mathrm{I}_{2} \mathrm{NO}_{4}$ : C, 36.03; H, 1.69; N, 2.63; found: $\mathrm{C}, 36.05 ; \mathrm{H}$, 1.68; N, 2.63; IR (KBr, cm $\left.{ }^{-1}\right): 3217(\mathrm{NH}, \mathrm{OH}), 1720(\mathrm{CO})$; ${ }^{1} \mathrm{H}$ NMR (300 MHz, DMSO- $\left.d_{6}, \delta / \mathrm{ppm}\right) 7.25-7.85$ (m, 4H, Ar-H), 8.35 (d, $J=1.8 \mathrm{~Hz}, 1 \mathrm{H}$, Ar-H-7), 8.43 (d, $J=1.8 \mathrm{~Hz}$, 1H, Ar-H-5), 8.70 (s, 1H, H-4), 10.12 (brs, 1H, OH), 11.9 (brs, $1 \mathrm{H}, \mathrm{NH}) ;{ }^{13} \mathrm{C}$ NMR $\left(75 \mathrm{MHz}, \mathrm{DMSO}-d_{6}, \delta / \mathrm{ppm}\right) 89.5$ (C-6), 92.0 (C-8), 130.5, 124.4, 134.3, 121.5 (C-3',4',5',6'), 114.3, 140.8, 116.0 (C-3,1',2'), 125.5 (C-4a), 134.2 (C-5), 139.5 (C-4), 144.5 (C-7), 146.6 (C-8a), 160 (CO $\delta$ lactone), $163.2(\mathrm{CONH})$, $170(\mathrm{COOH}) ; \mathrm{MS} m / z$ (\% relative intensity): $533\left(\mathrm{M}^{+}, 45\right), 425$ $\left(\mathrm{M}^{+}-\mathrm{NH}-\mathrm{C}_{6} \mathrm{H}_{4}-\mathrm{OH}, 100\right), 341$ (16), 214 (10), 171 (17), 87 (63).

2-(6,8-Diiodocoumarin-3-carboxamido)benzoic acid (10). Yellow crystals: Yield 91\%; mp $315{ }^{\circ} \mathrm{C}$; Anal. calcd for $\mathrm{C}_{17} \mathrm{H}_{9} \mathrm{I}_{2} \mathrm{NO}_{5}$ : C, 36.37; H, 1.60; N, 2.50; found: C, 36.39; $\mathrm{H}$, 36.39; N, 2.52; IR (KBr, cm $\left.{ }^{-1}\right): 3271(\mathrm{OH}, \mathrm{NH}), 3055(\mathrm{Ar}-\mathrm{H})$, 1751, 1651 (CO, CONH); ${ }^{1} \mathrm{H}$ NMR (300 MHz, DMSO- $d_{6}, \delta /$ ppm) 7.27, 7.64 (2d, $J=8.4 \mathrm{~Hz}, 4 \mathrm{H}, \mathrm{AB}-\mathrm{q}, \mathrm{Ar}-\mathrm{H}), 8.38$ (d, $J=$ $1.8 \mathrm{~Hz}, 1 \mathrm{H}$, Ar-H-7), 8.47 (d, $J=1.8 \mathrm{~Hz}, 1 \mathrm{H}, \mathrm{Ar}-\mathrm{H}-5), 8.71$ (s, $1 \mathrm{H}, \mathrm{H}-4), 10.10$ (brs, $1 \mathrm{H}, \mathrm{NH}), 10.49$ (s, $1 \mathrm{H}, \mathrm{OH}) ;{ }^{13} \mathrm{C} \mathrm{NMR}$ (75 MHz, DMSO- $\left.d_{6}, \delta / \mathrm{ppm}\right) 90.0$ (C-6), 92.1 (C-8), 123.0, 130.0 (C-2',3',5',6'), 114.3, 135.0, 130.5 (C-3,1',4'), 125.5 (C-4a), 133.2 (C-5), 138.5 (C-4), 144.2 (C-7), 146.1 (C-8a), 160 (CO $\delta$ lactone), 163.4 (CO-amide), $176.5(\mathrm{COOH}) ; \mathrm{MS} \mathrm{m} / \mathrm{z}(\%$ relative intensity): $561\left(\mathrm{M}^{+}, 7.1\right), 560(\mathrm{M}-1,40.8), 517\left(\mathrm{M}^{+}{ }_{-}\right.$ $\left.\mathrm{CO}_{2}, 2.7\right), 516\left(\mathrm{M}-\mathrm{CO}_{2} \mathrm{H}, 24.5\right), 425\left(\mathrm{M}-\mathrm{NHC}_{6} \mathrm{H}_{4}-2-\mathrm{CO}_{2} \mathrm{H}\right.$, 11.5), 424 (100), 341 (17), 171 (14.5) and 87 (58.2).

\section{2-(4-(6,8-Diiodocoumarin-3-carboxamido)phenyl)acetic acid} (11). Yellow crystals: Yield 93\%; mp $285{ }^{\circ} \mathrm{C}$; Anal. calcd for $\mathrm{C}_{18} \mathrm{H}_{11} \mathrm{I}_{2} \mathrm{NO}_{5}$ : $\mathrm{C}, 37.57$;, 1.91 ; N, 2.44; found: $\mathrm{C}, 37.59$; $\mathrm{H}$, 1.92; N, 2.46; IR (KBr, cm $\left.{ }^{-1}\right): 3286(\mathrm{OH}, \mathrm{NH}), 3047$ (Ar-H), 2916 (aliphatic-H), 1720 (CO); ${ }^{1} \mathrm{H}$ NMR (300 MHz, DMSO- $d_{6}$, $\delta / \mathrm{ppm}) 3.55$ (s, 2H, $\left.\mathrm{CH}_{2}\right), 7.27,7.64(2 \mathrm{~d}, J=8.4 \mathrm{~Hz}, 4 \mathrm{H}, \mathrm{AB}-\mathrm{q}$, 
Ar-H), 8.38 (d, $J=1.8 \mathrm{~Hz}, 1 \mathrm{H}$, Ar-H-7), 8.47 (d, $J=1.8 \mathrm{~Hz}$, 1H, Ar-H-5), 8.71 (s, 1H, H-4), 10.10 (brs, 1H, NH), 10.49 (s, $1 \mathrm{H}, \mathrm{OH}) ;{ }^{13} \mathrm{C}$ NMR $\left(75 \mathrm{MHz}, \mathrm{DMSO}-d_{6}, \delta / \mathrm{ppm}\right) 90.0$ (C-6), 92.1 (C-8), 123.0, $130.0\left(\mathrm{C}^{\prime}, 3^{\prime}, 5^{\prime}, 6^{\prime}\right), 114.3,135.0,130.5$ (C-3,1',4'), 125.5 (C-4a), 133.2 (C-5), 138.5 (C-4), 144.2 (C-7), 146.1 (C-8a), 160 (CO $\delta$ lactone), 163.4 (CO-amide), 176.5 $(\mathrm{COOH}) ; \mathrm{MS} m / z$ (\% relative intensity): $575\left(\mathrm{M}^{+}, 12.4\right), 574$ $(\mathrm{M}-1,68.9), 531\left(\mathrm{M}-\mathrm{CO}_{2}, 7.3\right), 425\left(\mathrm{M}-\mathrm{NH}_{-} \mathrm{C}_{6} \mathrm{H}_{4}-4-\right.$ $\left.\mathrm{CH}_{2} \mathrm{COOH}, 13.5\right), 424$ (100), 341 (18.9), 171 (27.2), 106 (94.6) and 87 (59.5).

\section{General procedure for the synthesis of ethyl cyanoacetate and pyridine derivatives 12 and 13}

Ethanolic solution of ethyl 6,8-diiodocoumarin-3-carboxylate (1) $(0.47 \mathrm{~g}, 10 \mathrm{mmol}, 30 \mathrm{~mL})$ was refluxed with ethyl cyanoacetate $(1.13 \mathrm{~g}, 10 \mathrm{mmol})$ for $6 \mathrm{~h}$. The solid precipitated was filtered off while hot, washed with ethanol and dried under vacuum, and was identified as compound $\mathbf{1 2}$. The filtrate evaporated under reduced pressure to produce a solid identified as compound 13. Compound 12 crystallized from dioxane, whereas compound $\mathbf{1 3}$ crystallized from chloroform.

Ethyl 2-(3-carbamoyl-6,8-diiodocoumarin-4-yl)-2-cyanoacetate (12). Pale yellow crystal: Yield $82 \%$; mp $310{ }^{\circ} \mathrm{C}$; Anal. calcd for $\mathrm{C}_{15} \mathrm{H}_{10} \mathrm{I}_{2} \mathrm{~N}_{2} \mathrm{O}_{5}: \mathrm{C}, 32.62 ; \mathrm{H}, 1.81 ; \mathrm{N}, 5.07$; found: $\mathrm{C}$, 32.64; H, 1.79; N, 5.05; IR ( $\left.\mathrm{KBr}, \mathrm{cm}^{-1}\right): 3309,3277\left(\mathrm{NH}_{2}\right)$, $2206(\mathrm{CN}), 1643(\mathrm{CO}) ;{ }^{1} \mathrm{H}$ NMR (300 MHz, $\left.\mathrm{CDCl}_{3}, \delta / \mathrm{ppm}\right)$ $1.50\left(\mathrm{t}, J=7.2 \mathrm{~Hz}, 3 \mathrm{H}, \mathrm{CH}_{3}\right), 4.44\left(\mathrm{q}, J=7.2 \mathrm{~Hz}, 2 \mathrm{H}, \mathrm{CH}_{2}\right)$, $5.05(\mathrm{~s}, 1 \mathrm{H}, \mathrm{CH}), 8.00(\mathrm{~d}, J=1.8 \mathrm{~Hz}, 1 \mathrm{H}, \mathrm{Ar}-\mathrm{H}-7), 8.40$ (d, $J=$ $1.8 \mathrm{~Hz}, 1 \mathrm{H}, \mathrm{Ar}-\mathrm{H}-5), 8.70$ (brs, $2 \mathrm{H}, \mathrm{NH}_{2}$, exchangeable with $\mathrm{D}_{2} \mathrm{O}$ ); $\mathrm{MS} m / z$ (\% relative intensity): $552\left(\mathrm{M}^{+}, 2\right), 388(8.0)$, 313 (30.0), 264 (4.0), 236 (35.0).

2-Amino-4-hydroxy-7,9-diiodocoumarino[3,4-c]pyridine-1carbonitrile (13). Pale yellow crystals: Yield $84 \%$; mp $340{ }^{\circ} \mathrm{C}$; Anal. calcd for $\mathrm{C}_{13} \mathrm{H}_{5} \mathrm{I}_{2} \mathrm{~N}_{3} \mathrm{O}_{3}: \mathrm{C}, 30.90 ; \mathrm{H}, 0.99 ; \mathrm{N}, 8.32$; found C, 30.92; H, 1.00; N, 8.34; IR (KBr, cm $\left.{ }^{-1}\right): 3374(\mathrm{OH}), 3277$, $3228\left(\mathrm{NH}_{2}\right), 2207(\mathrm{CN}), 1707,1662(\mathrm{CO}) ;{ }^{1} \mathrm{H} \mathrm{NMR}(300 \mathrm{MHz}$, $\left.\mathrm{CDCl}_{3}, \delta / \mathrm{pm}\right) 9.06$ (brs, $1 \mathrm{H}, \mathrm{OH}$, exchangeable with $\mathrm{D}_{2} \mathrm{O}$ ), 8.20 (s, 1H, H-8), 7.97 (s, 1H, H-10), 7.89 (brs, 2H, $\mathrm{NH}_{2}$, exchangeable with $\left.\mathrm{D}_{2} \mathrm{O}\right)$; $\mathrm{MS} m / z$ (\% relative intensity): $505\left(\mathrm{M}^{+}, 100\right)$, 477 (M - CO, 18.9), 397 (20.8), 341 (18.9), 171 (25.2), 106 (32.6) and 87 (35.5).

\section{General procedure for the synthesis of 1,3- oxazocine-5-carboxylate derivatives $14 a, b$}

A mixture of compound 1 (2.35 g, $5 \mathrm{mmol})$, acetone $(30 \mathrm{~mL})$ and (a) ammonium acetate $(0.4 \mathrm{~g}, 5 \mathrm{mmol})$ or (b) methylamine $(0.16 \mathrm{~g}, 5 \mathrm{mmol})$ was stirred at room temperature for 7 days. In both cases a colorless solid formed after the solvent had evapo- rated under reduced pressure, and the products were identified as compounds $\mathbf{1 4 a}$ and $\mathbf{1 4 b}$. The crude products were crystallized from benzene.

Ethyl 4-oxo-2,6-methano-2-methyl-3,4,5,6-tetrahydro-8,10diiodobenzo[2,1-g]-2H-1,3-oxazocine-5-carboxylate (14a). Colorless: Yield 72\%; mp $222{ }^{\circ} \mathrm{C}$; Anal. calcd for $\mathrm{C}_{15} \mathrm{H}_{15} \mathrm{I}_{2} \mathrm{NO}_{4}$ : C, 34.16; H, 2.85; N, 2.66; found: C, 34.18; H, 2.87; N, 2.68; IR (KBr, cm $\left.{ }^{-1}\right): 3217(\mathrm{NH}), 2977$ (aliphatic-H), 1728, 1689 (CO); ${ }^{1} \mathrm{H}$ NMR (300 MHz, DMSO- $\left.d_{6}, \delta / \mathrm{ppm}\right) 0.87$ $\left(\mathrm{t}, J=6.9 \mathrm{~Hz}, 3 \mathrm{H}, \mathrm{CH}_{3}(\mathrm{e})\right), 1.64-1.95\left(\mathrm{~m}, 5 \mathrm{H}, \mathrm{CH}_{2}(\mathrm{c})\right.$, $\left.\mathrm{CH}_{3}(\mathrm{f})\right), 3.80$ (q, $\left.J=4.5 \mathrm{~Hz}, 2 \mathrm{H}, \mathrm{CH}_{2}(\mathrm{~d})\right), 3.72-3.97$ (m, 2H, $\mathrm{H}(\mathrm{a})+\mathrm{H}(\mathrm{b})), 7.16$ (d, $J=1.8 \mathrm{~Hz}, 1 \mathrm{H}, \mathrm{Ar}-\mathrm{H}-9), 7.92$ (d, $J=2.1$ $\mathrm{Hz}, 1 \mathrm{H}, \mathrm{Ar}-\mathrm{H}-7), 8.88$ (brs, $1 \mathrm{H}, \mathrm{NH}) ;{ }^{13} \mathrm{C} \mathrm{NMR}(75 \mathrm{MHz}$, DMSO- $\left.d_{6}, \delta / \mathrm{ppm}\right) 14.1\left(\mathrm{CH}_{3}(\mathrm{e})\right), 24.7\left(\mathrm{CH}_{3}(\mathrm{f})\right), 42.5\left(\mathrm{CH}_{2}(\mathrm{c})\right)$, 56.0, $57.3(\mathrm{CH}(\mathrm{a})-\mathrm{CH}(\mathrm{b})), 61.2\left(\mathrm{CH}_{2}(\mathrm{~d})\right), 130,138,141.9,154$ (C-2,3,5), 87.1, 87.5 (C-4,6), 168.4 (CONH), 170 (CO); MS m/z (\% relative intensity): $527\left(\mathrm{M}^{+}, 4.3\right) 454\left(\mathrm{M}-\mathrm{CO}_{2} \mathrm{C}_{2} \mathrm{H}_{5}, 42.5\right)$, 182 (100), 136 (44.8), 57 (13.4).

Ethyl 3-methyl-4-oxo-2,6-methano-2,3-dimethyl-3,4,5,6tetrahydro-8,10-diiodobenzo[2,1-g]-2H-1,3-oxazocine-5carboxylate (14b). Colorless: Yield $70 \%$; mp $198{ }^{\circ} \mathrm{C}$; Anal. calcd for $\mathrm{C}_{16} \mathrm{H}_{17} \mathrm{I}_{2} \mathrm{NO}_{4}$ : C, 35.50; H, 3.14; N, 2.59; found: C, 35.51; H, 3.16; N, 2.61; IR (KBr, cm $\left.{ }^{-1}\right)$ : 3051 (Ar-H), 2985.6 (aliphatic-H), 1735, 1651 (CO); ${ }^{1} \mathrm{H}$ NMR (300 MHz, DMSO$\left.d_{6}, \delta / \mathrm{ppm}\right) 1.23\left(\mathrm{t}, J=7.2 \mathrm{~Hz}, 3 \mathrm{H}, \mathrm{CH}_{3}(\mathrm{e})\right), 1.78(\mathrm{~s}, 3 \mathrm{H}$, $\left.\mathrm{CH}_{3}(\mathrm{f})\right), 2.83$ (s, 3H, $\left.\mathrm{NCH}_{3}\right), 2.38-2.42\left(\mathrm{~m}, 2 \mathrm{H}, \mathrm{CH}_{2}(\mathrm{~b})\right)$, 3.4-3.57 (m, 2H, H(a) + H(b)), 4.18 (q, $J=7.2 \mathrm{~Hz}, 2 \mathrm{H}$, $\left.\mathrm{CH}_{2}(\mathrm{~d})\right), 7.66$ (d, 1H, Ar-H-9), 7.95 (d, $J=1.8 \mathrm{~Hz}, 1 \mathrm{H}, \mathrm{Ar}-\mathrm{H}-$ 7); MS $m / z$ (\% relative intensity): $541\left(\mathrm{M}^{+}, 3.4\right), 196$ (59.2), 150 (27.6), 56 (100).

\section{Antimicrobial assays}

The newly synthesized compounds were screened for their antimicrobial activities in vitro against two species of Gram-positive bacteria, namely Staphylococcus aureus (NCTC-7447), Bacillus cereus (ATCC-14579), and two Gram-negative bacteria, namely Escherichia coli (NCTC-10410), Serratia marcescens (IMRU-70); and against two species of fungi, namely Aspergillus fumigatus (MTCC-3008) and Candida albicans (MTCC-227). The tested microorganisms were obtained from the Regional Center for Mycology \& Biotechnology (RCMP), Al-Azhar University.

The activities of these compounds were tested by using the discdiffusion method [23] for bacteria and the paper-disk-diffusion method [24] for fungi. The area of zone inhibition was measured with ampicillin $\left(30 \mu \mathrm{g} \mathrm{mL}^{-1}\right)$ as the standard antibiotic reference for antibacterial activity, and calforan $\left(30 \mu \mathrm{g} \mathrm{mL}^{-1}\right)$ was used as a reference antifungal activity. The 
tested compounds were dissolved in $N, N$-dimethylformamide (DMF) to give a solution of $1 \mathrm{mg} \mathrm{mL}{ }^{-1}$. The inhibition zones (diameter of the hole) were measured in millimeters $(6 \mathrm{~mm})$ at the end of an incubation period of $48 \mathrm{~h}$ at $28^{\circ} \mathrm{C} ; N, N$-dimethylformamide showed no inhibition zone.

\section{Conclusion}

It was interesting to note that four of the new compounds (12, 13 and 14a,b) were found to have an antimicrobial activity greater than that of the standard antibiotic ampicillin or the standard antifungal claforan, while compounds 1-11 were either inactive or only weakly active against the tested microorganisms. The presence of fused diiodocoumarino[3,4-c]pyridine and diiodobenzo[2,1-g]-2H-1,3-oxazocine nucleus increased the antimicrobial activity, whereas the presence of diiodocoumarin3 -carboxamides decreased the antimicrobial activity.

\section{References}

1. O'Kennedy, R.; Thornes, R. D. Coumarins: Biology, Applications and Mode of Action; Wiley \& Sons: Chichester, UK, 1997.

2. Zahradnik, M. The Production and Application of Fluorescent Brightening Agents; Wiley \& Sons, 1992.

3. Bailly, C.; Bal, C.; Barbier, P.; Combes, S.; Finet, J.-P.; Hildebrand, M.-P.; Peyrot, V.; Wattez, N. J. Med. Chem. 2003, 46, 5437-5444. doi:10.1021/jm030903d

4. Yeh, J.-Y.; Coumar, M. S.; Horng, J.-T.; Shiao, H.-Y.; Kuo, F.-M.; Lee, H.-L.; Chen, I.-C.; Chang, C.-W.; Tang, W.-F.; Tseng, S.-N. J. Med. Chem. 2010, 53, 1519-1533. doi:10.1021/jm901570x

5. Pierson, J.-T.; Dumètre, A.; Hutter, S.; Delmas, F.; Laget, M.; Finet, J.-P.; Azas, N.; Combes, S. Eur. J. Med. Chem. 2010, 45, 864-869. doi:10.1016/j.ejmech.2009.10.022

6. Basile, A.; Sorbo, S.; Spadaro, V.; Bruno, M.; Maggio, A.; Faraone, N.; Rosselli, S. Molecules 2009, 14, 939-952. doi:10.3390/molecules14030939

7. Zhao, P.-L.; Wang, L.; Zhu, X.-L.; Huang, X.; Zhan, C.-G.; Wu, J.-W.; Yang, G.-F. J. Am. Chem. Soc. 2010, 132, 185-194. doi:10.1021/ja905756c

8. Teichert, J. F.; Feringa, B. L. Chem. Commun. 2011, 47, 2679-2681. doi:10.1039/c0cc05160h

9. Key, J. A.; Kho, S.; Timerghazin, Q. K.; Brown, A.; Cairo, C. W. Dyes Pigm. 2009, 82, 196-203. doi:10.1016/j.dyepig.2009.01.001

10. Zhou, S.; Jia, J.; Gao, J.; Han, L.; Li, Y.; Sheng, W. Dyes Pigm. 2010, 86, 123-128. doi:10.1016/j.dyepig.2009.12.005

11. Craven, P. A.; Pfanstiel, J.; DeRubertis, F. R. J. Clin. Invest. 1986, 77, 850-859. doi:10.1172/JCl112382

12. Rathbone, D. L.; Su, D.; Wang, Y.; Billington, D. C. Tetrahedron Lett. 2000, 41, 123-126. doi:10.1016/S0040-4039(99)02027-4

13. Mishra, J. K.; Samanta, K.; Jain, M.; Dikshit, M.; Panda, G. Med. Chem. Lett. 2010, 20, 244-247. doi:10.1016/j.bmcl.2009.10.126

14. Burton, H. J. Chem. Soc. 1945, 280, 280-283. doi:10.1039/JR9450000280

15. Bonsignore, L.; Cottiglia, F.; Maccioni, A. M.; Sacci, D.; Lavagna, S. M. J. Heterocycl. Chem. 1995, 32, 573-577. doi:10.1002/jhet.5570320234

16. Koelsch, C. F. J. Am. Chem. Soc. 1945, 67, 569-574. doi:10.1021/ja01220a023
17. Koelsch, C. F.; Freerks, M. C. J. Org. Chem. 1953, 18, 1538-1545. doi:10.1021/jo50017a013

18. Bedair, A. H.; Aly, F. M.; El-Agrody, A. M.; El-Assy, R. K. M. Acta Pharm. 1986, 36, 363-369.

19. Kadnikov, D. V.; Larock, R. C. J. Organomet. Chem. 2003, 687, 425-435. doi:10.1016/S0022-328X(03)00786-1

20. Potdar, M. K.; Mohile, S. S.; Salunkhe, M. M. Tetrahedron Lett. 2001 42, 9285-9287. doi:10.1016/S0040-4039(01)02041-X

21. Yavari, I.; Adib, M.; Hojabri, L. Tetrahedron 2002, 58, 6895-6899. doi:10.1016/S0040-4020(02)00758-5

22. Creaven, B. S.; Egan, D. A.; Kavanagh, K.; McCann, M.; Noble, A.; Thati, B.; Walsh, M. Inorg. Chim. Acta 2006, 359, 3976-3984. doi:10.1016/j.ica.2006.04.006

23. European Committee for Antimicrobial Susceptibility Testing (EUCAST) of the European Society of Clinical Microbiology and Infectious Diseases (ESCMID). Clin. Microbiol. Infect. 2000, 6, 509-515.

24. National Committee for Clinical Laboratory Standards. Methods for dilution antimicrobial susceptibility tests for bacteria that grow aerobically, 5th ed; Approved Standard M7-A5.Wayne, PA: NCCLS, 2000

\section{License and Terms}

This is an Open Access article under the terms of the Creative Commons Attribution License (http://creativecommons.org/licenses/by/2.0), which permits unrestricted use, distribution, and reproduction in any medium, provided the original work is properly cited.

The license is subject to the Beilstein Journal of Organic Chemistry terms and conditions: (http://www.beilstein-journals.org/bjoc)

The definitive version of this article is the electronic one which can be found at: doi:10.3762/bjoc.7.199 